$\begin{array}{cl}\begin{array}{c}\text { Revue } \\ \text { de } / \text { histoire }\end{array} & \text { Revue de l'histoire des religions } \\ \text { des religions } & \begin{array}{l}3 \mid 2010 \\ \text { Varia }\end{array}\end{array}$

\title{
Pontoise, la Ligue et le Carmel : la mystique au service de l'union politique autour de l'année 1605
}

Pontoise, the League and the Carmelites: Mysticism in the Service of the Political Union, ca. 1605

\section{Stéphane-Marie Morgain}

\section{OpenEdition}

\section{Journals}

Édition électronique

URL : http://journals.openedition.org/rhr/7621

DOI : $10.4000 /$ rhr.762

ISSN : 2105-2573

Éditeur

Armand Colin

Édition imprimée

Date de publication : 1 octobre 2010

Pagination : 335-354

ISBN : 978-2200-92657-1

ISSN : 0035-1423

\section{Référence électronique}

Stéphane-Marie Morgain, « Pontoise, la Lique et le Carmel : la mystique au service de l'union politique autour de l'année 1605 », Revue de l'histoire des religions [En ligne], 3 | 2010, mis en ligne le 01 octobre 2013, consulté le 30 avril 2019. URL : http://journals.openedition.org/rhr/7621 ; DOI : 10.4000/

rhr.7621

Ce document a été généré automatiquement le 30 avril 2019

Tous droits réservés 


\section{Pontoise, la Ligue et le Carmel : la mystique au service de l'union politique autour de l'année 1605}

Pontoise, the League and the Carmelites: Mysticism in the Service of the Political Union, ca. 1605

Stéphane-Marie Morgain

1 Lorsqu'en 1587 le cordelier Noël Taillepied ${ }^{1}$ publie à Rouen ses Antiquitez et singularitez de la ville de Pontoise ${ }^{2}$, la capitale du Vexin français, engagée résolument dans le camp de la Ligue, est sur le point d'affronter une défaite militaire majeure qui sera l'origine d'un déclin démographique, économique et social, mais aussi celle d'un profond renouveau du catholicisme. La fondation à Pontoise, le 16 janvier 1605, du second carmel de la réforme thérésienne en France, témoigne effectivement des nouvelles aspirations spirituelles d'une ville meurtrie par ses luttes intestines. Il convient ainsi de resituer cet établissement dans son contexte pour en saisir toute la pertinence.

Ce ne sont pas directement les péripéties de l'installation à Pontoise des carmélites venues de Paris, ni la haute personnalité des premières moniales qui motivent ces pages, d'autres historiens l'ont fait avec compétence ${ }^{3}$. C'est plutôt le récit des événements liés au combat des Pontoisiens ligueurs et leur soumission finale à Henri IV qui nous conduira à l'identification des membres de l'élite catholique, ouvrière de l'arrivée des filles de sainte Thérèse et de leur implantation.

3 Pontoise, ville du Vexin français, appartient au gouvernement de l'île-de-France. À quelque six lieues de Paris, elle domine le confluent de la Viosne et de l'Oise. Meulan, Magny, Chaumont, Mante, Poissy, Saint-Germain finissent de composer cette région riche en blés et en vignobles ${ }^{4}$. La situation géographique de cette cité, passage important sur l'Oise et route directe de Paris à la mer, attire l'attention du stratège et attise les convoitises des belligérants. Les ligueurs sauront en tirer profit durant les guerres civiles 
du XVI ${ }^{\mathrm{e}}$ siècle, comme l'avait fait Charles VII en 1441 lors de la reprise de la ville aux Anglais au terme de six semaines de furieux combats.

4 Au début du règne de Charles IX, alors que les temps sont plutôt mauvais pour le catholicisme, les États généraux d'Orléans de décembre 1560 convoquent de nouveaux États à Pontoise pour le mois d'août de l'année suivante. Le $1^{\mathrm{er}}$ août, l'assemblée peu nombreuse - treize députés de la noblesse et autant du Tiers État ; le clergé est à Poissy ${ }^{5}$ - siège dans la grande salle du couvent des cordeliers. Elle demande la convocation des États tous les deux ans, la liberté religieuse, la réunion d'un concile national, la réforme du clergé, l'unité dans la justice, le vote de l'impôt, l'interdiction de toute guerre offensive sans l'assentiment des États. Mais surtout, les députés proposent de dépouiller le clergé de la totalité de ses biens pour éteindre la dette de l'État et alléger certaines impositions. Prévoyant, avisé, ou tout simplement affolé, le clergé s'empresse de faire un don de 15 millions à la couronne. Une séance plénière réunie à Saint-Germain le 27 août conclut cette Assemblée.

5 Les positions des députés du Tiers ne reflètent pas pour autant celles de la population pontoisienne dévouée aux intérêts catholiques et ennemie déclarée de la "religion prétendue réformée ». Ce sont assurément ces sentiments anti-protestants qui dirigeront la ville vers le parti de la Ligue. L'exemple du procès fait à Jean Boicervoise, lieutenant civil et criminel du bailli de Senlis à Pontoise depuis 1548, illustre bien cet état d'esprit. Une déclaration royale du 27 janvier 1562 "pour entretenir le peuple en paix et concorde ", autorise les protestants à tenir, de jour, en dehors de l'enceinte des villes, des réunions où se feraient leurs prêches et leurs prières. Or, Boicervoise, Charles Chouart, prévôt et garde des Sceaux de la châtellenie, et l'avocat du roi David Aubery ${ }^{6}$, pratiquaient le calvinisme en pleine ville et se promettaient d'ériger un temple à Pontoise. De violentes émeutes éclatèrent instantanément dans les rues. En réaction, le corps de ville décida de poursuivre Boicervoise devant le Parlement de Paris et le 23 juillet le lieutenant du bailli est condamné à être pendu sur la place de Grève. Pour interrompre durablement les troubles, l'exercice public du culte réformé, pour le ressort du bailliage de Senlis, est transféré de Pontoise à Verberie ${ }^{7}$. Cet éloignement forcé des huguenots de Pontoise exprime la nette aversion de la population pour le calvinisme.

Noël Taillepied corrobore ce fort attachement au catholicisme : « Le peuple de Pontoise est tant dévot envers Dieu, qu'on ne verra jamais hommes vagabonds parmi les rues et aux tavernes pendant qu'on dira vêpres, la messe ou durant un sermon [...] On n'oserait aussi se promener dans les églises durant qu'on y chante le service divin sous peine d'être puni ${ }^{8} »$.

7 Ces quelques éléments laissent entrevoir les raisons du basculement de Pontoise du côté de la Ligue au moment du soulèvement de Paris contre Henri III.

\section{L'entrée de Pontoise dans sa période militante}

8 La victoire d'Henri de Navarre à Coutras le 20 octobre 1587, qui anéantit en deux heures l'armée catholique conduite par l'impatient duc de Joyeuse, attisa la colère des ligueurs parisiens, précipita l'humiliation d'Henri III et renforça dangereusement la puissance des Guise.

9 Le 13 mai 1588, au lendemain de la célèbre "journée des barricades ", qui consacrait l'arrogante popularité d'Henri de Guise, le roi quitte Paris pour Chartres abandonnant la 
capitale au "balafré ». La veille, le roi avait appelé en renfort le régiment de Picardie, mais les Pontoisiens lui avaient barré le passage du pont signifiant ainsi leur opposition au souverain et leur soutien aux ligueurs. Cette action, fatale au monarque, était lourde de sens politique.

10 Après sa fuite, Henri III se résigne finalement à négocier avec les ligueurs. Il signe le 15 juillet à Rouen l'édit d'Union, qui confirme celui de Nemours de juillet 1585. Il renouvelle le serment du sacre d'extirper l'hérésie, «sans faire jamais aucune paix ou trêve avec les hérétiques ni aucun édit en leur faveur ${ }^{9}$ ", scelle son union avec la Ligue, amnistie les ligueurs pour leurs exactions des 12 et 13 mai et nomme Henri de Guise lieutenant général des armées. Le coup porté à l'autorité de Valois est sévère et doit le ramener à la raison.

11 Le 8 septembre, au début des États généraux réunis à Blois, Henri III, à la surprise générale, change brutalement toute l'équipe ministérielle qui l'avait poussé à négocier avec Guise. Le chancelier Philippe Hurault de Cheverny, le surintendant Pomponne de Bellièvre, les secrétaires d'État Nicolas de Villeroy, Nicolas Brûlart et Claude Pinart sont renvoyés ; François de Montholon est chargé des sceaux, Martin Ruzé de Beaulieu et Louis de Revol remplacent les secrétaires d'État. Si l'ambassadeur espagnol, Mendoza, s'étonne de cette décision prise sans l'avis de Catherine de Médicis ni celui du duc de Guise, Étienne Pasquier note au contraire que voulant plaire au peuple et aux députés des États généraux, le roi affiche ici son désir d'inaugurer une ère nouvelle ${ }^{10}$.

12 De fait, incapable de maîtriser la hardiesse croissante des demandes du Tiers, qu'il croit manipulée par les Guise, Henri III décide de la façon la plus désespérée et la plus hasardeuse d'en finir ${ }^{11}$. Le 23 décembre, il fait assassiner Henri de Guise et le jour suivant son frère Louis, cardinal archevêque de Rouen, dont Sixte-Quint disait méchamment qu'il n'avait de cardinal que le bonnet. Ce « coup de majesté », selon l'expression d'Yves-Marie Bercé $^{12}$, n'est pas un assassinat, c'est une exécution décidée pour le bien de l'État et le maintient de l'autorité monarchique. La reine mère, atterrée, ne peut plus rien. Elle meurt d'ailleurs le 5 janvier suivant. Henri III pensait avoir sauvé son trône et raffermi son pouvoir en éliminant le chef charismatique de la Ligue. « Il pensait avoir tué la Ligue. En fait, elle commençait ${ }^{13}$ ».

13 Le souverain avait donc congédié les États de Blois: Orléans, Rouen, Amiens, Beauvais, Pontoise, Péronne, Montdidier, Clermont, Beaumont-sur-Oise et d'autres villes se prononcent pour la Ligue et s'attachent à Charles de Mayenne, dernier frère d'Henri et Louis de Guise, qui avait évité le massacre, à Charles d'Aumale, son cousin germain, et à d'autres seigneurs évêques.

Bien que piètre chef de guerre, comme le prouveront ses défaites d'Arques et d'Ivry en 1589 et 1590 contre Henri IV, Mayenne est proclamé par le conseil des Seize, « lieutenant général de l'Estat royal et Couronne de France », en « attendant l'assemblée des Estats du royaume ». Il place ses garnisons dans les villes qui lui sont favorables, comme Pontoise, et se prépare à un combat qui allait inévitablement éclater. Aumale nommé gouverneur de Paris, installe les autres gouverneurs promus par la Ligue : il commença par Pontoise « vers laquelle ville il prit sa brisée pour en faire estat et y mettre gens à sa dévotion pour y tenir ferme ${ }^{14} »$.

15 Pour sortir d'une crise dans laquelle il s'embourbait et malgré ses répugnances naturelles à s'allier avec les réformés, Henri III signe le 3 avril 1589 un traité avec les émissaires du roi de Navarre. Le 30 du même mois a lieu à Plessis-lès-Tours une entrevue entre les deux 
hommes. Henri de Bourbon plia le genou devant le roi qui le releva en l'appelant son frère : le geste théâtral et imposé scellait l'alliance.

Début juin, l'armée royale commence sa marche vers Paris. Elle occupe successivement tous les passages de la Loire, à l'exception d'Orléans et de Nantes. Depuis Chartres, où Henri III reçut la fausse assurance de Claude Vatherie, procureur-syndic de Pontoise que la ville ne prendrait pas de garnison de la Ligue, l'armée s'avança vers Poissy et Pontoise pour assurer la possession des rives de l'Oise et de la Seine. Le 30 juillet, l'armée est devant Paris et le roi s'installe au château de Saint-Cloud. À ce moment, Henri III croit proche sa victoire sur la capitale.

\section{Les officiers pontoisiens à la veille du siège de la ville}

À cette date, le lieutenant général du bailli de Senlis, en résidence à Pontoise est Jacques de Monthiers. Il avait épousé par contrat le 17 septembre 1559, Marguerite Dauvergne ${ }^{15}$. Leur fils, Gabriel de Monthiers, seigneur de Saint-Martin, est prévôt-maire de la ville avant de succéder à son père en 1594. Le procureur du roi, André Fournier est marchand bourgeois, et le maître de l'écurie se nomme Jacques Gérard. Les échevins sont Robert François, avocat, et Guillaume Dancongnée, marchand. Leurs successeurs en octobre 1592 seront Simon Charton et Antoine Maître, marchand, et en 1596, Pierre Duval, procureur, frère d'André Duval, le célèbre professeur titulaire depuis 1597 d'une des deux chaires de théologie de la Sorbonne, et fils de Robert Duval - qui en 1562 était allé à Moulins plaider la cause catholique devant Charles IX -, et Jacques Foubert, marchand. Enfin, l'argentier de la ville s'appelle Jehan Legros.

Jacques de La Saussaye, grand-vicaire de Pontoise, représente l'archevêque de Rouen, Charles de Bourbon. Le gouverneur de la ville est Georges Babou, seigneur de la Bourdaisière, comte de Sagonne, frère d'Isabeau de Sourdis, oncle de Gabrielle d'Estrée, premier gentilhomme du duc d'Alençon et ancien chef des ligueurs chartrains ${ }^{16}$.

Mais c'est surtout la figure de Charles de Neufville de Villeroy, de Magny, de Parmes, de Bury, de la Forêt Thaumier, etc., connu sous le nom de d'Alincourt ou d'Halincourt qui domine l'histoire de la Ligue à Pontoise. Né en 1566, il est l'unique fils de Nicolas IV de Neufville de Villeroy et de Madeleine de L'Aubespine ${ }^{17}$. Entré rapidement dans la carrière des armes, il sert sous le commandement du maréchal Jacques de Matignon pendant sa campagne avec Mayenne contre l'armée du roi de Navarre. À la suite de la prise de SainteBazeille, de Montaigu et de Castillon, il porte la cornette blanche de son régiment. Villeroy qui a pour son fils les ambitions les plus hautes, le fait entrer au service d'Anne de Joyeuse qui le nomme guidon de sa compagnie de gendarmes. Aux côtés du favori d'Henri III, Halincourt prend part à la défaite de Coutras le 20 octobre 1587 et le mois suivant, rejoint le duc d'Éperon qui, en Bourgogne, pourchasse les survivants de l'armée protestante battus à Auneau le 24 novembre par le duc de Guise.

Le 28 février 1588, à 22 ans, il épouse Marguerite de Mandelot, dame de Passy et de Lerné, fille de François de Mandelot, gouverneur de Lyon depuis février $1571^{18}$, et d'Éléonore de Robertet $^{19}$. Cette alliance devait légitimement assurer à Charles d'Halincourt le gouvernement du Lyonnais et maintenir la ville dans l'obéissance royale ${ }^{20}$. Le roi désirait fortement détourner Mandelot de la Ligue. Mais dès l'automne 1582, il projette de le destituer au profit de Bernard de La Valette. Les relations entre les deux hommes vont se détériorer dès qu'Épernon convoitera ouvertement la citadelle de la ville. En juillet 1584, 
le roi songe à confier le gouvernement de Lyon à Henri de Joyeuse, comte du Bouchage, et le commandement de la citadelle au cousin d'Épernon, Philippe-Antoine de Moncassin. Le 5 mai 1585, furieux de l'amputation de son autorité sur la citadelle, Mandelot laisse la population s'en emparer avant de la raser. Ni le roi, ni Épernon ne lui pardonneront cette insolente conduite. Nicolas de Villeroy interviendra pour obtenir le pardon royal. Pendant l'été, Épernon décide de prendre le commandement de l'armée du Dauphiné. En juillet de l'année suivante, il mandera son oncle le baron de Termes et Philibert de La Guiche pour négocier le passage dans Lyon ${ }^{21}$. En juin 1586, la rumeur court que Mandelot est mort : le gouvernement est accordé à Bernard de La Vallette, et le mois suivant il est à nouveau promis à Joyeuse.

21 Les événements parisiens de mai 1588 ébranlent les certitudes des Villeroy quant à l'obtention du gouvernement lyonnais. D'autant que durant l'été, Catherine de Médicis travaille avec la duchesse de Nemours à obtenir pour le jeune duc la survivance de ce gouvernement dont Jacques de Savoie avait jadis assuré la charge. L'imposition faite à Villeroy de quitter la cour le 23 août achève de ruiner les espérances d'Halincourt. La mort prématurée de François de Mandelot, le 24 novembre 1588, y mettra un point final.

Malgré la requête des membres du consulat de confier le gouvernement à Halincourt « comme estant déjà bien versé au manyement de la charge » et « bien zelé et affectionné à la religion catholique, apostolique et romaine, au service de Sa Majesté et repos de la ville $^{22}$ », Henri III nomme Charles-Emmanuel de Nemours et, de surcroît, prive le gendre de Mandelot de la lieutenance générale qu'il confie au sénéchal Guillaume de Gadagne, alors chargé de l'intérim. Le nouveau gouverneur ne vient pas immédiatement prendre ses fonctions car depuis le 23 décembre, il est emprisonné à Blois avec sa mère, le cardinal de Guise et l'archevêque de Lyon Henri d'Épinac. Le 23 février 1589, lors d'une nouvelle journée des barricades, Lyon se soulève contre l'autorité royale et rejoint les rangs de la Sainte-Union. Halincourt est-il responsable de ce basculement? C'est bien probable. Toujours est-il que le 12 mars, Mayenne lui confie la lieutenance générale ôtée au royaliste Gadagne. Nemours entre dans Lyon le 22 mars. Les échevins l'accueillent en lui proposant une sorte de contrat par lequel il doit s'engager à respecter les privilèges de la ville $^{23}$. L'opposition de plus en plus vive des Lyonnais à l'égard d'Halincourt le pousse à quitter la province pour rejoindre sa ville de Pontoise qu'il atteint en juin $1589^{24}$.

À Pontoise il est assisté par Edme de Hautefort, seigneur de Thenon, second fils de Jean de Hautefort et de Catherine de Chabannes. Gentilhomme de la chambre du roi, lié aux Guise et à Tavannes ${ }^{25}$, gouverneur et sénéchal du Limousin en juin $1580^{26}$, lieutenant général au gouvernement d'Auvergne, puis de Champagne et de Brie pour la Ligue, il avait été membre du conseil général de la Sainte-Union. Plus âgé que d'Halincourt, plus résolu aussi, il servira sous ses ordres. Halincourt est aussi secondé par un corps spécial de Lyonnais qu'il a ramené de ses précédentes mésaventure ${ }^{27}$ et par Monsieur de Serans et le chevalier de Flavacourt, parent du bailli de Gisors.

\section{Le siège de Pontoise}

Les troupes régulières de la Ligue de Pontoise se composent de deux mille hommes, d'un corps de lansquenets et de cinq cents chevaux, auxquels viennent s'ajouter deux mille hommes supplémentaires envoyés en renfort par Mayenne ${ }^{28}$. Ce nombre semblait insuffisant puisque Villeroy envoya Antoine Esgret et Nicolas Souvoye demander d'autres troupes à Charles de Lorraine. Fortifiée, armée, munie de vivre, vidée de certains 
bourgeois qui avaient fui vers Paris, Pontoise pouvait résister longtemps aux armées royales.

Après avoir facilement pris Poissy, les troupes d'Henri III sont accrues par celles du duc de Montpensier arrivées de Picardie et de Normandie. Maître du cours de la Seine, il fallait aussi le devenir du passage de l'Oise. La conquête de Pontoise est donc absolument indispensable. Les hommes du roi de Navarre se mettent en marche avec le duc d'Éperon et le maréchal de Biron. Un pont sur l'Oise est construit par les Suisses rendant le passage plus facile. Éperon s'avance vers le faubourg Notre-Dame, Navarre contourne Pontoise par la vallée de la Viosne, Henri III établit son quartier général à Cergy. Les troupes sont en vue vers le 8 ou le 9 juillet. Le siège commence immédiatement, il est partiellement relaté dans Le Discours du siège de Pontoise, contenant ce qui s'est passé depuis l'unzième de juillet jusques à présent, publié à Paris chez Pierre Des-Hayes en 1589 par un ligueur convaincu ${ }^{29}$.

À partir du mardi 11 juillet, Pontoise est assiégée par les deux Henri, la garnison commandée par Halincourt est soumise à de terribles assauts qui ont promptement raison des défenses de la ville malgré l'héroïque résistance des ligueurs comme en témoignera Henri IV dans une lettre du 14 juillet à sa maitresse la comtesse de Gramont ${ }^{30}$. Le 12, le combat est encore plus rude. La panique s'empare des habitants et des soldats qui se précipitent vers la porte de Paris pour fuir. Le chevalier de Flavacourt fait incontinent fermer les issues et en remet les clefs à Monthiers. Le même jour, Edme de Hautefort est tué d'un coup d'arquebuse en pleine tête. "Une perte pour la Ligue » avoue Henri de Navarre qui avait lui-même perdu beaucoup de ses gentilshommes. Le 17, tandis que la lutte se poursuit avec la même vigueur, Halincourt est blessé au bras (ou à l'épaule) d'un coup d'arquebuse ${ }^{31}$. Il n'est plus capable de diriger les opérations de défense. L'église et le bastion autour de Notre-Dame sont en ruines, le faubourg en flammes, la muraille prête à céder. Jean de Testefort élève son héros en chef vertueux et lucide, se soumettant à l'ordre de la nécessité plutôt que de « ruiner une ville et grande ville en luy résistant avec des forces inesgales et disproportionnées ${ }^{32}$ ».

Le 23 juillet, on pense à parlementer et à capituler. Le 24 , une reddition de la ville est négociée. Navarre se montra plus compréhensif qu'Henri III qui avait fait pendre les chefs de la Ligue à Pithiviers et à Étampes. Les ligueurs pontoisiens sont émus par l'humanité d'Henri de Navarre. Le 25 juillet, Pontoise est soumise à une très forte contribution de guerre pour le prix de la libre sortie des troupes ligueuses désarmées ${ }^{33}$. D'abord fixée à cent mille écus, ce qui aurait ruiné la ville, la contribution passa soixante mille écus puis enfin à quarante-cinq mille écus ${ }^{34}$. C'est à Nicolas Fournier, à qui Noël Taillepied avait dédié ses Antiquitez de Pontoise, qu'est confiée la mission d'établir les comptes des recettes et du paiement de cette imposition. Le travail sera achevé par sa veuve ${ }^{35}$. Le $1^{\text {er }}$ août, à Saint-Cloud, Henri III tombe sous le couteau du moine Jacques Clément. Le roi meurt le lendemain. Après la Déclaration du 4 août, par laquelle Henri IV promet le maintien de la religion catholique, s'engage à se soumettre à un concile national ou général et à convoquer les États généraux dans les six mois, environ la moitié de la France le reconnait comme roi. Parmi les villes de la région parisienne, il faut citer Pontoise, Melun, Meulan et Compiègne.

28 À la fin du mois de décembre 1589, après dix-sept jours de siège, la ville est reprise par les ligueurs qui suivent Jean de Saulx, seigneur de Tavannes ${ }^{36}$. La citadelle ne capitulera que le 6 janvier $1590^{37}$. Le gouvernement de la ville est à nouveau confié à Halincourt. Si Mayenne souhaitait le récompenser pour son courage durant le siège de 1589, il voulait 
surtout engager son père à rester à Paris dans le camp ligueur ${ }^{38}$. Après la bataille d'Ivry du 14 mars 1590 remportée par les neuf mille hommes commandés par Henri IV sur les quinze mille ligueurs du duc de Mayenne, Pontoise reste la seule ville ligueuse au milieu d'une région passée aux royalistes.

Au début de 1591, Pontoise est de nouveau menacée par un siège. Malgré l'annonce de renforts du côté de Mayenne ${ }^{39}$ et de quelques petites actions d'Halincourt, dont la prise de l'Isle Adam le 21 août qui met Henri IV dans une violente colère ${ }^{40}$, la ville est de nouveau perdue pour la Ligue le 12 septembre ${ }^{41}$.

1592 marque le début de claires négociations entre Mayenne et Henri IV sur les instances de Villeroy. Si celles de mai 1592 échouèrent, Nicolas de Villeroy obtint une trêve de sept mois pour la région de Pontoise et du Vexin français. Le $1^{\mathrm{er}}$ septembre, à Meulan, son fils la signa au nom du duc de Mayenne, avec François d'O, gouverneur de l'île de France, afin «de parvenir au soulagement du peuple, faciliter la cueillette des fruictz estans sur la terre, le moyen de faire les semences pour la présente année et donner moyen au peuple de vivre ${ }^{42}$ ». Cette trêve, ratifiée par Henri IV le 30 septembre est reconduite pour un an en mars 1593. Ce nouveau délai coïncide pratiquement avec l'entrée d'Henri IV à Paris (22 mars) et la remise de Pontoise sous son obéissance. Mais pour le moment, il semble encore que d'Halincourt poursuive son combat aux côtés de la Ligue ${ }^{43}$.

31 Son père, au contraire, est acquis à Henri IV au début de 1594. Il obtient de Bellièvre et de Zamet une nouvelle trêve de trois mois pour convaincre son fils d'ouvrir au souverain les portes de Pontoise. Ce qu'il fera le 20 ou 22 mars 1594. Le ralliement des Villeroy à la couronne a un prix. Dès le mois de février Pierre de l'Estoile notait dans son Journal : « Au commencement de ce mois, le sieur de Villeroy ayant fait son accord et celui de son fils, le sieur d'Halincourt, avec le Roy pour la ville de Pontoise, est rentré au service de Sa Majesté, qui lui a donné l'employ de secrétaire d'État, qu'il avait occupé sous Henri III ${ }^{44}$ ». De fait, Nicolas de Neufville retrouvera sa charge de secrétaire d'État en septembre de la même année, à la mort de Louis de Revol. Les motifs du rapprochement sont donc parfaitement intéressés et politiques. Ils sont aussi financiers puisque Villeroy reçoit une indemnité de 476594 livres. La capitulation de Pontoise coûtait cher au trésor royal ${ }^{45}$. Henri IV avait raison de dire qu'on lui avait vendu et bien vendu la ville. Il apparaît donc quelque peu exagéré de penser que les catholiques pontoisiens se soient jetés aux pieds du roi aussitôt après son abjuration, entraînant leur gouverneur ligueur dans leur mouvement d'enthousiasme.

Charles d'Halincourt, quant à lui, est désormais libre de poursuivre sa carrière et de briguer les charges les plus prestigieuses, ce qu'il ne manquera pas de faire.

Le 10 juillet 1593, il avait perdu sa femme, Marguerite Mandelot, âgée de vingt-trois ans. Elle avait été enterrée au couvent des cordeliers de Pontoise et laissait deux filles vivantes ${ }^{46}$. Le 11 février 1596, Halincourt épousera Jacqueline de Harlay, fille de Nicolas de Harlay, baron de Sancy, premier maître d'hôtel du roi, colonel général des Suisses ${ }^{47}$. Moins d'un mois plus tard Henri IV nommait le jeune homme " gentilhomme de la chambre du roi ${ }^{48}$ ».

$\mathrm{Au}$ sortir de la crise ligueuse, Pontoise est en ruine, endettée, appauvrie et sa dépopulation amorcée avec la guerre de Cent ans, s'accélère : quatre mille à cinq mille habitants vers 1600 contre dix mille au début du XIV siècle. Face à la centralisation croissante qui tend à éclipser progressivement le rayonnement de la ville et la priver de son autonomie en la plaçant dans l'orbite de Paris, deux pôles tentent de résister; l'aristocratie fieffée aux alentours de Pontoise et attachée à ses terres : Andrésy, Puiseux, 
Boisemont, Contenaut, Mouy, Commery, Boury, Halincourt ; et la bourgeoisie des offices au service des juridictions royales et des élites municipales ${ }^{49}$.

\section{Un carmel pontoisien} carmélites à Pontoise. Le choix de Pontoise comme lieu pour la fondation du second carmel de France est d'abord le fait de sa proximité de Paris. Ainsi, la Mère Anne de Jésus (de Lobéra), prieure du monastère de Paris et les trois supérieurs pourraient-ils visiter facilement le nouveau couvent. Mais on objecta que la ville, quoique fort pieuse, était aussi «très petite et pauvre" et "on craignait que les religieuses ne manquassent d'assistance pour les secours spirituels - les jésuites ${ }^{50}$ et les capucins n'étant pas encore établis - et d'ailleurs personne ne se présentait pour fonder le monastère ${ }^{51}$ ». L'idée émanait d'André Duval nommé en novembre 1603 par Clément VIII, supérieur des carmélites de France en même temps que Jacques Gallemant, curé d'Aumale, et Pierre de Bérulle. Natif de Pontoise, son frère Pierre, procureur du roi avait été nommé échevin en 1596, et son père Robert, avait accompagné en 1562 la délégation mandatée à Moulins auprès de Charles IX. André Duval était bien placé pour plaider en faveur d'une cité dont il connaissait la piété et l'attachement à la religion catholique. Pour emporter l'adhésion des plus récalcitrants, il proposa une maison rue du Soleil ou de l'Épée (aujourd'hui rue Marcel-Rousier) qu'il avait achetée le 11 septembre 1602 à Marie de Charron ${ }^{52}$.

Barbe Acarie avait acquis la protection de Michel de Marillac et obtenu de son amie Marie de Bréauté (Charlotte de Harlay de Sancy, veuve du marquis de Bréauté), alors novice au carmel de Paris, et belle-sœur de Charles d'Halincourt, qu'elle constitue une rente suffisante " pour porter le titre de fondatrice du couvent de Pontoise $\mathrm{e}^{53}$ ».

Parmi les premières novices auxquelles Jacques Gallemant avait donné une formation depuis 1599, il faut relever Agnès de Jésus Deslions (ou Des Lions), née à Pontoise le 17 juillet 1580, fille de François Deslions, lieutenant des Eaux et Forêts de Pontoise, maître des caves, seigneur de Thieuville, et d'Agnès Fournier; Marie de Jésus (Nicole Lefèvre), née à Pontoise vers 1583, fille de Nicolas Lefèvre, avocat au Parlement; Marie de SaintJoseph (Nicole Fournier), née à Pontoise le 3 novembre 1584. Elle était la petite-fille de Guillaume Fournier, "gouverneur de villes » au temps des troubles, et la fille de Nicolas Fournier, marchand drapier, "homme honorable et estimé de ses concitoyens » à qui Noël Taillepied avez dédié ses Antiquitez en $1587^{54}$, et de Marie Cossart, - on se souvient que c'est à Nicolas Fournier qu'avait été confiée la charge d'établir les comptes des recettes et du paiement de la taxe de 45000 écus imposée par Henri IV à la ville -; et Françoise de la Croix (Françoise Charton), née à Pontoise en 1575 ou 1577, fille de Maître Charton, officier du roi, demeurant à Pontoise. Toutes quatre prendront l'habit le 17 janvier dans le nouveau carmel fondé deux jours plus tôt.

Pour composer les bâtiments de la nouvelle implantation il fallut ajouter à la maison d'André Duval, celle de Marie Cossart, veuve de Nicolas Fournier et mère de sœur Marie de Saint-Joseph ${ }^{55}$. D'autres bâtisses et jardins seront acquis par la suite. L'une à Jehan Langlois qui l'avait achetée à Pierre de La Cour le 11 avril 1603, et une autre aux marguilliers de Saint-Maclou. Le 25 juillet 1605, Geneviève Marcassin, veuve de Jean d'Orvalle " contrôleur au magasin et grenier à sel de Ponthoise ", donne aux carmélites sa maison de la rue de l'Épée " pour être assocyée à leurs prières et ensemble ses parens et amys tant vivans que trespassés ${ }^{56}$ ». Enfin, une dernière maison est léguée par Marguerite 
Dauvergne, épouse de Jacques de Monthiers et mère de Gabriel de Monthiers, lieutenant général du bailli de Senlis en 1594 .

Mais les carmélites ne pouvant obtenir la propriété du puits qui était en commun avec le presbytère de Saint-Maclou, décident de s'établir en bas de la ville ${ }^{57}$. La maison de Geneviève Marcassin est vendue le 11 septembre 1608 à Denise Quentin, veuve de Pierre de Vannes, Cécile Oudun et Madeleine Tellier, le bâtiment contigu, acquis par procuration aux héritiers de Nicolas Groslay le 3 et 11 janvier 1605, est échangé le 23 juin 1606 contre une maison rue de la Grande-Tannerie, appartenant à maître Nicolas Honoré, qui avait fui Pontoise pendant le siège "comme traître et politique ${ }^{58}$ ». Une fois la communauté installée en son nouveau lieu, trois maisons sont revendues à Jean Langlois, le 3 décembre 1610 et la quatrième le sera quelque temps plus tard ${ }^{59}$.

Un incontestable courage, voire une véritable audace, étaient indispensables aux catholiques dévots pour inviter des carmélites déchaussées espagnoles à instaurer un monastère cloîtré dans une ville marquée par les séquelles des récents conflits et par la pression financière. La mitoyenneté des deux anciennes citées ligueuses joua certainement un rôle déterminant dans la décision des fondateurs. De même, la tradition authentiquement catholique de Pontoise et son opposition proverbiale au calvinisme déterminèrent un choix politique que renforçait encore l'autorité morale d'André Duval.

41 Toutes ces raisons, avouées ou non, sont fort judicieuses et confirment le bon sens du groupe Acarie et des Mères espagnoles. Mais le succès de l'entreprise est-il seulement la conséquence d'un projet minutieusement pensé ? Le lien entre Pontoise, la Ligue et le Carmel, s'épuise-t-il dans l'énonciation d'une procession litanique? Il existe, nous semble-t-il, une certaine communication entre ces termes. Ce qui convoque cette hypothèse, c'est la correspondance immédiate entre le milieu ligueur - ou compris comme tel -, très actif durant les sièges de Pontoise entre 1589 et 1594 et la société catholique à l'origine de l'établissement des filles de sainte Thérèse en janvier 1605 . L'énumération des familles d'officiers pontoisiens alliés au gouvernement de Charles d'Halincourt, celle des fondateurs, des bienfaiteurs et des premières novices renforce cette conjecture.

42 Sans mettre en cause la sincérité religieuse et spirituelle du groupe, ne peut-on pas imaginer, comme cela se vérifie par ailleurs pour d'autres implantations carmélitaines ${ }^{60}$, que l'établissement à Pontoise d'un Ordre né de la réforme catholique offrait à ces familles une aubaine pour racheter aux yeux du Très Chrétien leur ancien comportement rebelle ? Cette conversion de la Ligue au Carmel, de la lutte à la soumission au roi, suscite cette dépendance des termes et donne aux ouvriers d'une pieuse opération, de reconquérir une virginité perdue dans le lit de la désobéissance.

Le Carmel apparaît ici encore comme un lieu capable de rassembler autour d'un même idéal spirituel et mystique des élites naturellement opposées politiquement et qui le resteront encore pendant quelques décennies. Les anciens ligueurs, ou les «cryptoligueurs ", peuvent collaborer avec des catholiques royaux à la mise en place d'un projet commun de réforme de l'Église. L'Ordre de la sainte d'Avila canalise et purifie ainsi les forces et les pulsions d'un renouveau tant politique que religieux.

Le passage des Fournier, des Lefèvre, des Monthiers, des Duval, des Cossart et des Dauvergne, d'un comportement de guerre à une attitude pacifique, d'une lutte armée contre le souverain terrestre, à une subordination sans faille au lieutenant du roi céleste, manifeste donc la bonne compréhension que cette élite sociale avait du Carmel thérésien, 
dont l'oraison mentale et affective constituait un lieu privilégié de réconciliation politique et de silence mystique.

\section{NOTES}

1. Pierre Moracchini, « Noël Tallepied (1540-1589) », Entretiens autour de Noël Taillepied, Mémoires de la société historique et archéologique de Pontoise, du Val-d'Oise et du Vexin, t. LXXVI, 1990-1991, p. 399-418.

2. Noël Taillepied, Les Antiquitez et singularitez de la ville de Pontoise, Rouen, impr. de Georges L'Oiselet, 1587 ; Rouen, R. Du Petit Val, 1587 (édition revue et annotée sur les manuscrits des archives de Pontoise et collationnée sur l'imprimé de 1587 par A. François, précédée d'une notice biographique et bibliographique sur l'auteur par Henri Le Charpentier... [et suivie d'un appendice contenant des extraits de notes de M. Pihan de La Forest], Pontoise, Alexandre Seyès, 1876).

3. Chroniques de l'ordre des carmélites de la réforme de sainte Thérèse depuis leur introduction en France, vol. I, Troyes, 1846, p. 124-133 ; Marcel Rigal, Le carmel de Pontoise, Lisieux, 1960 ; Philippe Ferry, «Pontoise, le plus ancien carmel de France», Vivre en Val d'Oise, n 1, avril 1990, p. 37-43 ; JeanDominique Mellot, Histoire du carmel de Pontoise, t. I, (1605-1792), Paris, Desclée de Brouwer, 1994.

4. Louis Duval, Abrégé des Antiquitez de la ville de Pontoise et des personnes illustres de ladite ville, Rouen, P.-P. Cabut, 1720 ; Abbé Trou, Recherches historiques, archéologiques et biographiques sur la ville de Pontoise, Pontoise, impr. de Dufey, 1841 (rééd. Marseille, 1977) ; James Gressier, JeanMarcel Champion, Alain Demurger et al., Pontoise 2000 ans d'histoire, Pontoise, 1973.

5. L'assemblée des évêques s'était ouverte le 31 juillet. Le colloque se tiendra entre le 9 septembre et le 14 octobre.

6. Sur la famille Aubery, son ascension et ses alliances avec les Bellièvre, voir Olivier Poncet, Pomponne de Bellièvre (1529-1607). Un homme d'État au temps des guerres de religion, Paris, Mémoires et documents de l'École des Chartes ( $\left.n^{\circ} 50\right)$, 1998. La mère de Marie de La Trinité d'Hannivel, première professe du carmel français s'appelait Marie Aubery, fille de Claude Aubery, seigneur de Trilleport-lès-Meaux (Stéphane-Marie Morgain, «Marie de La Trinité d'Hannivel. Première professe du carmel de France (1579-1647) », Carmel, n 111, mars 2004, p. 93-110).

7. Henri Le Charpentier, La Ligue à Pontoise et dans le Vexin français. Recherches historiques et archéologiques sur la fin $d u x I^{e}$ siècle, Pontoise, Alexandre Seyès, 1878, p. 21-23. La Ligue dans le Vexin normand. Journal d'un bourgeois de Gisors, relation historique concernant les événements accomplis $\grave{a}$ Paris et dans les environs, et notamment dans et entre les villes de Rouen, Amiens, Beauvais, Pontoise (1588-1617), Paris, éd. Henri Le Charpentier et A. Fitan, 1878.

8. Noël Taillepied, op. cit., $38 \mathrm{r}^{\circ}-\mathrm{v}^{\circ}$.

9. Eugène et Émile Haag, La France protestante, Paris, J. Cherbuliez, 1846-1859, vol. 10, p. 201-203.

10. Jean-Marie Constant, La Ligue, Paris, Fayard, 1996, p.184-185 ; Arlette Jouanna, («Les ambiguïtés des Politiques face à la Sainte Ligue », De Michel de L'Hospital à l'Édit de Nantes. Politique et religion face aux Églises, Thierry Wanegffelen (dir.), Clermont-Ferrand, Presses universitaires Blaise-Pascal, 2002, p. 475-493), montre que les prises de positions très favorables à la journée des barricades faites par les Politiques (Étienne Pasquier, Jean Bodin ou Guillaume Du Vair), pourraient remettre en cause l'idée courante qu'ils constituent des défenseurs inconditionnels de l'autorité légitime. Voir l'ouvrage fondamental de Robert Descimon, Qui étaient les Seize? Mythes et 
réalités de la Ligue parisienne (1585-1594), Paris, Fédération des sociétés historiques et archéologiques de Paris et de l'île de France, 1983.

11. Jean-Marie Constant, Les Guise, Paris, Fayard, 1984, p. 226 ; La Ligue, op. cit., p. 206-212.

12. Yves-Marie Bercé, "Les coups de majesté ", Complots et conjurations dans l'Europe moderne, Yves-Marie Bercé et Elena Fasano Guarini (dir.), Rome, École française de Rome, 1996, p. 491-505.

13. Jean-Marie Constant, La Ligue, op. cit., p. 212.

14. Chronique de Jehan Mallet, citée par Henri Le Charpentier, op. cit., p. 47.

15. Henri Le Charpentier (ibid., p. 64) indique par erreur Marguerite Davergne.

16. Nicolas Le Roux, La Faveur du roi. Mignon et courtisans au temps des derniers Valois (vers 1547-vers 1589), Paris, Champ Vallon, 2000, p. 56, 194, 310.

17. Jean Testefort, Livre premier de la parfaicte idée d'un bon Gouverneur prise sur Monseigneur d'Halincourt. Par un Religieux Docteur de l'Ordre de St Dominique, 1642. Yann Lignereux, Lyon et le roi. De la « bonne ville » à l'absolutisme municipal (1594-1654), Paris, Champ Vallon, 2003, p. 263-270.

18. Il avait été nommé par Catherine de Médicis durant les dernières années du règne de Charles IX.

19. Antoine Péricaud, Notice sur François de Mandelot, gouverneur et lieutenant-général du Lyonnais, Forez et Beaujolais sous Charles IX et Henri III, [Avec un post-scriptum signé : B. Chevalier-Victor], Extrait des « Archives historiques du Rhône », Lyon, J.-M. Barret, 1828.

20. Dès le 2 juillet 1587, le roi signait une promesse accordant le gouvernement du Lyonnais, Forez et Beaujolais à Halincourt en cas de mariage avec la fille de Mandelot. La survivance de ce gouvernement est expédiée et scellée en forme de provisions par Pierre Brûlart le 11 mars 1588. (Yann Lignereux, Lyon et le roi, p. 236).

21. Dans une lettre adressée à Villeroy le 10 août 1586, Mandelot écrit : « Il ne tiendra en moy que Monsieur d'Espernon ne se loue de l'accueil qui luy aura esté faict en ceste ville, car nous scavons toutz que le Roy l'ayme, et comme ses très-fidelles subjectz et serviteurs doibvent aymer et honnorer tout ce qui est aymé de sadite Mejesté, pour mon particulier je feray ce que je doibtz », cité par Nicolas Le Roux, op. cit., p. 562.

22. Cité par Yann Lignereux, op. cit., p. 239.

23. Jean Henri Mariéjol, Charles Emmanuel de Savoie, duc de Nemours, Paris, Hachette, 1938, p. 87.

24. Henri Le Charpentier, op. cit., p. 68, n. 1.

25. Nicolas Le Roux, op. cit., p. 85.

26. Ibid., p. 331. En juin 1588, Henri III le destituera de sa charge, avant de décréter son arrestation après la mort des Guise (ibid., p. 691).

27. La chanson nouvelle où est descrite la vertu et la valeur des Lyonnais dans la défense de Pontoise, sur le chant "Estendu parmy les fleurs », éditée par E. Bonnejoy, Paris, 1873. Le texte est reproduit dans Henri Le Charpentier, op. cit., p. XI-XVI.

28. On lira avec plaisir le récit romancé qu'Henri Le Charpentier publia en 1861 dans l'Écho pontoisien («Claire et Gaétan. Épisode du siège de Pontoise par Henri III », Euvres de Henri Le Charpentier. Mélanges historiques sur Pontoise, Pontoise, 1886, p. 1-50).

29. Le texte est retranscrit dans Henri Le Charpentier, op. cit., p. I-IV.

30. Ibid., p. LXXVI-LXXVII.

31. L'auteur du Brief discours de la défaicte de l'armée de Henry de Bourbon, jadis roi de Navarre, devant la ville de Pontoise, avec le nombre de morts, faicte par M. le duc de Mayenne, et autres seigneurs, unis pour la deffence de la foi Chrestienne, Catholique, Apostolique et Romaine (A Tolose, par Jacques Colomiez, 1589) se montre bien optimiste puisque la ville tombera quelques jours après (Henri Le Charpentier, op. cit., p. v-VIII).

32. Yann Lignereux, op. cit., p. 267.

33. Henri Le Charpentier, op. cit., p. 116.

34. Mémoires authentiques de Jacques Nompar de Caumont, duc de La Force, maréchal de France, et de ses deux fils, les marquis de Montpouillan et de Castelnaut, éd. Marquis de La Grange, Paris, 1843, t. 1, 
p. 62-63. Le 29 mars 1594, les habitants de Pontoise adresseront à Henri IV une supplique pour lui demander une diminution des impôts, « en considérations des grandes pertes et ruines qu'ils ont soufferts dans les deux sièges, nourriture des garnisons et grandes dettes de toutes sortes dont ils sont chargés ".

35. Extrait du Compte rendu de l'assiette de 45000 écus de contribution imposée à la ville de Pontoise, après le siège, en 1589, Henri Le Charpentier, op. cit., p. XVII-XX.

36. Henri Le Charpentier (ibid., p. 154) confond Jean et Gaspard de Saulx-Tavannes. Gaspard de Tavannes, père de Jean, meurt en 1573. Jean (1555-1629) fait imprimer en 1623 ou 1624 ses Mémoires sous le nom de Gaspard de Saulx, maréchal de Tavannes. Ce qui explique l'erreur de Le Charpentier. Jean de Saulx-Tavannes obtiendra de bien modestes conditions de reddition. Il se soumettra le 14 juin 1595 contre 9000 écus et la promesse de la dignité de maréchal, qu'il attendra toute sa vie. Modeste, par rapport aux 3766825 livres versées à Charles de Lorraine et aux 900000 livres de Claude de La Châtre en plus du maintien de sa dignité de maréchal et de ses gouvernements de l'Orléanais et du Berry.

37. La Reprinse de la ville de Ponthoise et du chasteau de Vincennes faictes nouvellement par monsieur de duc de Mayenne, lieutenant général de l'Estat et Couronne de France, ensemble les enseignes qui ont esté en l'église de Notstre-Dame à Paris, Paris, 1590.

38. Le 12 juillet 1589, Nicolas de Villeroy avait quitté Pontoise. "Voyant l'investissement de la place, il se retirait à Paris, vers le duc de Mayenne ", Henri Le Charpentier, op. cit., p. 90. «Villeroy avait fait paraître en décembre 1589 un Avis d'État sur les affaires de ce temps, par lequel il invitait Mayenne à reconnaître Henri IV en le sommant d'abjurer. Le 26 mars 1590, il rencontre Mornay et Biron ; le 13 avril, Henri IV reçoit lui-même Villeroy dans les faubourgs de Melun, mais les Espagnols empêchent Mayenne de négocier et Villeroy apparaît de plus en plus suspect à ces derniers » (Yann Lignereux, op. cit., p. 267-268, n. 6 et Jean-Pierre Babelon, Henri IV, Paris, Fayard, 1982, p. 488).

39. Henri Le Charpentier, op. cit., p. 177-178.

40. Ibid., p. 182-183.

41. À la même date, Sully tenta de s'emparer de Pontoise en tendant un piège à Halincourt et à Mayenne centré sur Mantes (Bernard Barbiche et Ségolène de Dainville-Barbiche, Sully, Paris, 1997, p. 48, et La Trahison découverte en la ville de Ponthoise avec l'exécution qu'on a facit des traistres et de la conversion de l'un d'iceux qui estoit hérétique, Paris, chez Robert Nivelle et Rolin Thierry, imprimeurs de la Sainte-Union, 1591).

42. Cité par Nicolas Le Roux, op. cit., p. 710. L'accord sera ratifié par Henri IV, au camp de Champs-sur-Marne, le 30 septembre 1592. Traité de 1592 entre le sieur d'O et le sieur d'Halincourt, Henri Le Charpentier, op. cit., p. XLV-XLVI.

43. Henri Le Charpentier, op. cit., p. 190-191.

44. Cité par ibid., p. 202.

45. Le ralliement de tous les chefs ligueurs, à l'exception de Mercœur qui résiste encore en Bretagne, aura coûté à Henri IV trente-deux millions de livres.

46. Un premier enfant, Charles de Neufville, né à Pontoise, était mort en 1592. Les deux filles sont: Madeleine qui épousera le marquis de Sillery et mourra sans descendance ; Catherine, dame d'honneur d'Anne d'Autriche, épousera Jean II de Souvré et mourra en 1657.

47. Six enfants naîtront de cette union: Nicolas IV de Neufville, duc de Villeroy, maréchal de France et gouverneur de Louis XIV ; Henri, comte de Buhy, mort en 1628 ; Camille, né à Rome en 1606, futur archevêque de Lyon; Ferdinand, chevalier de Malte, évêque de Saint-Malo ; LyonFrançois, tué au siège de Turin en 1639 ; Marie de Neufville, morte en 1688.

48. Gouverneur de Lyon à la mort de Philibert de La Guiche le 14 juin 1607, il avait été ambassadeur extraordinaire à Rome en janvier 1600. Il mourra à Lyon le 18 janvier 1642 à soixante-seize ans. Voir Henri Le Charpentier, op. cit., p. 212-217; Yann Lignereux, op. cit., p. 271-288. 
49. Dominique Mellot, op. cit., p. 16.

50. Henri Le Charpentier, Les Jésuites à Pontoise, recherches sur leur établissement, leur résidence et leur expulsion de cette ville (1593-1762), Pontoise, impr. de A. Paris, 1880.

51. Histoire du carmel de Pontoise, t. I, p. 139, cité par Dominique Mellot, op. cit., p. 40-41.

52. Dominique Mellot, op. cit., p. 269.

53. Cité par Dominique Mellot, op. cit., p. 41.

54. Noël Taillepied, op. cit., Epistre, non paginée : «Toutefois le devoir que je doibs avec vous à ceste ville et pays qui m'a nourri, enseigné, logé par si long temps, et décoré par nos prédications, l'espace de sept à huit ans, me rend debteur de le réciproquer, en si peu qu'il m'est possible de faveur, et lui rendre l'un des petits moyens que j'ai reçeus de lui ; par le récit de ses antiquités et excellences, que je lui ay dressé du bout de ma plume, et en ay fait un petit livre que je présente et dédie à vos révérences, comme à l'un de mes meilleurs amis, et qui le mérite mieux, eu esgard à la race dont vous êtes issu. On cognait assez que vostre feu père, le sire Guillaume Fournier (que Dieu absolve), fut de son temps gouverneur de villes du temps des troubles, durant lesquels mania tant bien les affaires, qu'il préserva le pays et la ville du pillage, et de l'hérésie qui y commençoit à couver et pulluler. Et pource qu'on cognoist mieux le faict que je ne le pourrais ici descrire, je feray fin, suppliant Dieu le Créateur, vous conserver et garder en bonne prospérité, et vous aussi de prendre le petit livre d'un tel cœur qu'il vous est présenté par le moindre de vos Orateurs qui vous baise les mains. »

55. Par acte de donation, daté du mardi 18 janvier 1605, Marie Cossart abandonne aux carmélites de Pontoise « une maison, jardin et jeu de paume, parce que lesdites religieuses ont reçu et admis le iours d'hier Nicolle Fournier, fille de la donatrice et dudit feu Fournier». L'acte stipule expressément: «Si ladite Nicole Fournier ne se trouve bien audit couvent, Marillac s'oblige à payer à la donatrice 1500 livres pour la valeur du jeu de paume et lieux sus désignés » (Arch. du Val d'Oise, 2 E 12033).

56. Arch. Carmel de Pontoise, copie de l'acte de donation du 25 juillet 1605.

57. En effet, la maison de Marie Cossart était attenante : "d'un côté à la maison et couvent où sont à présent établies lesdites carmélites ; d'un autre côté à une maison appartenant à l'église St Maclou de Ponthoise en laquelle est à présent demeurant Me Antoine Dupuy, prêtre ; d'un bout par derrière au jardin d'une autre maison appartenant à ladite église » (Arch. du Val d'Oise, $2 \mathrm{E}$ 12033).

58. Analyse des pièces du procès intenté par Claude Vatherie, greffier en chef de la prévôté-mairie de Pontoise, aux échevins de cette ville, 1592-1596, Henri Le Charpentier, op. cit., p. LX.

59. Dominique Mellot, op. cit., p. 269.

60. Stéphane-Marie Morgain, «L'installation des Carmes déchaux à Toulouse en mars 1623 : De la Ligue au catholicisme royal », Revue d'histoire de l'Église de France, t. 89, $\mathrm{n}^{\circ} 223$, juillet-décembre 2003, p. 363-383.

\section{RÉSUMÉS}

Comme beaucoup d'Ordres religieux engagés dans la réforme catholique en France, le Carmel thérésien offre à ses protagonistes un espace libre où la mystique devient le point de ralliement des anciens ligueurs et des catholiques royaux. La collaboration des deux partis à un projet identique permet de montrer au roi une volonté commune de contribuer, par la fondation d'un 
Ordre venu d'Espagne, à l'œuvre de "réconciliation " nationale. La fondation du carmel de Pontoise en 1605 est significative d'un schéma souvent répété. La population pontoisienne, massivement catholique et anti-protestante, s'engouffre dans la Ligue avant de se soumettre à Henri IV au terme des sièges répétés.

Like many religious orders involved in the Catholic reformation in France, the Teresian Carmelite order offered a free space where mysticism became the rallying point of former League members and royal Catholics. The collaboration between the two parties on a common project made it possible to demonstrate to the King a common willingness to contribute, through the founding of a new order from Spain, to the effort of national 'reconciliation'. The founding of a Carmelite house in Pontoise in 1605 was representative of a frequently repeated schema. The population of Pontoise, massively Catholic and anti-Protestant, was engulfed in the League before submitting to Henry IV after repeated sieges.

\section{AUTEUR}

STÉPHANE-MARIE MORGAIN

Institut catholique de Toulouse

sm.morgain@ict-toulouse.asso.fr 